\title{
Medición Testicular en Sementales de Raza Caballo Chileno Enteros y Castrados Unilateralmente
}

\author{
Testicular Measures in Entire And Unilaterally Castrated Chilean Horse \\ Breed Stallions
Lisandro Muñoz-Alonzo ${ }^{1.3}$, Felipe Morales Quintana ${ }^{2}$, Reinaldo Ortiz Ramírez ${ }^{\mathbf{1}}$, Jaime Cruces Leal ${ }^{1}$, Mario Briones Luengo ${ }^{2}$, Fernando Saravia Ramos ${ }^{2}$

\section{Resumen}

El objetivo del presente estudio fue establecer el tamaño testicular y estimar la producción espermática diaria (PED) en sementales de raza Caballo Chileno enteros y castrados unilateralmente. Se estudiaron 61 sementales (31 con ambos testículos y 30 castrados unilateralmente), de 3 a 20 años de edad. Se midió el alto, ancho y largo de los testículos con un vernier escrotal, se calculó el volumen testicular (VT) y se estimó la PED utilizando las fórmulas propuestas por Sertich (2011). Las medidas testiculares en los sementales con ambos testículos fueron mayores a las esperadas y sin diferencia estadística entre testículos contralaterales en sementales enteros. La PED estimada en sementales con ambos testículos fue de $7.67 \pm 2.32 \times 10^{9}$ espermatozoides. En los sementales castrados unilateralmente no hubo diferencias de tamaño relacionadas con el testículo que mantenían, pero el alto, ancho y VT fueron mayores que en los sementales enteros $(\mathrm{p}<0.05)$. La PED estimada en sementales castrados unilateralmente fue de $5.13 \pm 1.84 \mathrm{x}$ $10^{9}$ espermatozoides, lo cual es $33 \%$ menor que en caballos enteros $(\mathrm{p}<0.0001)$. Los resultados permiten concluir que las medidas testiculares de los sementales de raza Caballo Chileno son mayores a las esperadas para su tamaño corporal.

Palabras clave: volumen testicular, producción espermática diaria, equino

\section{Abstract}

The aim of this study was to determine the testicular size and to estimate the daily sperm production (DSP) in entire and unilaterally castrated males of the Chilean Horse breed. Sixty one ( 31 entire and 30 unilaterally castrated) stallions, with ages between 3 and 20 years of age were studied. A scrotal caliper was used to measure height, width and length of testicles. Testicular volume (TV) was calculated and DSP was estimated using

\footnotetext{
${ }^{1}$ Departamento de Ciencias Clínicas, ${ }^{2}$ Departamento de Ciencias Pecuarias, Facultad de Ciencias Veterinarias, Universidad de Concepción, Concepción, Chile

${ }^{3}$ E-mail: lismunoz@udec.cl
}

Recibido: 19 de octubre de 2015

Aceptado para publicación: 5 de marzo de 2016 
formulae proposed by Sertich (2011). Testicular measures in entire stallions were larger than expected and without statistical difference between testicles. The estimated DSP in entire stallions was $7.67 \pm 2.32 \times 10^{9}$ spermatozoa. There were no differences in size related to the remaining testicle in unilaterally castrated stallions, but height, width and TV were larger than in entire stallions ( $\mathrm{p}<0.05)$. DSP in unilaterally castrated stallions was $5.13 \pm$ $1.84 \times 10^{9}$ spermatozoa, and it was a $33 \%$ lower compared to entire males ( $\left.\mathrm{p}<0.0001\right)$. Results obtained allow concluding that testicular measures in Chilean Horse stallions are larger than expected for their body size.

Key words: testicular volume, daily sperm production, equine

\section{INTRODUCCIÓN}

La raza Caballo Chileno existe oficialmente desde 1893 (Porte et al., 2000). Es una raza de registro cerrado permitiéndose inscribir únicamente a caballos de padres de esta raza (Arms, 2015). Es un caballo de contextura gruesa, con una altura a la cruz entre 1.31 y $1.49 \mathrm{~cm}$ en los machos, perímetro torácico entre 1.56 y $1.80 \mathrm{~cm}$ (García et al., 1997; Muñoz et al., 2012) y un peso promedio de $386 \mathrm{~kg}$ (García et al., 1997).

El principal uso de caballos de esta raza es en el rodeo chileno (García et al., 1997), deporte que se realiza dentro de un picadero circular con superficie de arena, en donde dos caballos y sus respectivos jinetes deben arrear un novillo realizando un movimiento lateral al galope, para luego uno de los caballos detener con el pecho al novillo presionándolo contra una superficie acolchada (Federación de Rodeo Chileno, 2015). A muchos de los sementales que participan en el rodeo se les realiza castración unilateral para mejorar el rendimiento deportivo, aduciendo que el movimiento lateral al galope provoca traumatismo en uno de los testículos. Muchos de estos machos castrados unilateralmente posteriormente son utilizados como reproductores.

Las medidas testiculares se pueden obtener con un pie de rey o vernier escrotal o mediante ultrasonido (Chenier, 2007; Blanchard et al., 2008; Sertich, 2011), sin encontrarse diferencias entre estos métodos (Love et al., 1991). Las medidas testiculares permiten determinar en forma indirecta la capacidad reproductiva potencial de un semental (Kavak et al., 2003; Chenier, 2007; Varner, 2011) estimando la producción espermática diaria (PED) (Love et al., 1991, 2007; Sertich, 2011; Stout y Colenbrander, 2011), y de esa forma el número potencial de yeguas que puede cubrir al día un semental (Blanchard et al., 2008).

Las medidas testiculares deben ser un factor importante de considerar al seleccionar un reproductor (Pickett et al., 1981; Stout y Colenbrander, 2011). Inicialmente se utilizaba el ancho escrotal para estimar la PED (Gebauer et al., 1974b; Thompson et al., 1979; Pickett et al., 1981), sin embargo, Love et al. (1990) propusieron una fórmula más exacta basada en el volumen testicular, la que ha sido ampliamente utilizada (Chenier, 2007; Love, 2007; Sertich, 2011; Varner, 2011).

Debido a que no existen estudios previos en relación al tamaño testicular en sementales de raza Caballo Chileno, el objetivo de este estudio fue establecer el tamaño testicular en sementales enteros y castrados unilateralmente y estimar su PED.

\section{Materiales Y Métodos}

El trabajo se realizó en criaderos de la Región del Biobío, Chile, durante la estación reproductiva (septiembre-diciembre de 2010). 
Se evaluaron 61 equinos (Equus caballus), sementales de raza Caballo Chileno, inscritos en el libro de registro de la raza. Los animales tenían entre 3 y 20 años de edad, estaban reproductivamente sanos y con condición corporal entre 3 y 3.5 en la clasificación de 0 a 5 propuesta por Carroll y Huntington (1988). De estos, 31 sementales presentaban ambos testículos y 30 estaban castrados unilateralmente (12 con el testículo derecho y 18 con el izquierdo). Todos se mantenían en pesebreras individuales por 14 a 23 horas diarias con cama de viruta o aserrín y el resto del tiempo permanecían amarrados fuera de la pesebrera o sueltos en un corral. Su alimentación se basaba en heno de alfalfa, avena en grano, concentrado en pellets, sal y agua.

Se llevaron registros individuales con el nombre, edad y localización de los testículos. Se descartaron signos de inflamación, adherencias o torsión testicular mediante palpación. Se midieron los testículos con un vernier escrotal (Mitutoyo® 532-120, Japón/ Brasil), siguiendo el procedimiento descrito por Chenier (2007); es decir, con una mano se empujó el testículo hacia abajo dentro del escroto y con la otra mano se manipuló el vernier tomando las medidas del alto, largo y ancho (en $\mathrm{cm}$ ) de cada testículo. Cada medida fue tomada tres veces por un único operador y se utilizó el promedio a fin de minimizar el error del operador.

El volumen testicular (VT) fue calculado en centímetros cúbicos, utilizando la fórmula propuesta por Love et al. (1990) y recomendada por Sertich (2011) para sementales de razas livianas: $\mathrm{VT}=0.523 \mathrm{x}$ alto $\mathrm{x}$ largo $\mathrm{x}$ ancho. Asimismo, se estimó el número de espermatozoides de la PED de cada semental utilizando la fórmula recomendada por Sertich (2011) para sementales de razas livianas, donde PED = [0.024(VTI+VTD)]-0.76. En este caso, VTI es el volumen testicular izquierdo y VTD el volumen testicular derecho. Asimismo, en los sementales enteros se calculó el volumen testicular combinado (VTC), sumando el volumen testicular de ambos testículos.
El procedimiento utilizado fue aprobado por el Comité de Ética de la Facultad de Ciencias Veterinarias de la Universidad de Concepción, Chile.

El análisis estadístico se basó en la determinación de los promedios de las medidas testiculares obtenidas. Para esto se utilizó la comparación de dos muestras independientes con la prueba de «t» de Student (Hernández et al., 2010) con un nivel de significancia de $\mathrm{p}<0.05$. Se evaluaron las diferencias entre testículos contralaterales, diferencias de tamaño testicular y PED entre sementales enteros y sementales castrados unilateralmente.

\section{Resultados}

En los cuadros 1 y 2 se muestran los promedios, desviaciones estándar y rangos de las medidas testiculares de los animales enteros y con castración unilateral, respectivamente. El VTC en los sementales enteros fue de $351.2 \pm 98.4 \mathrm{~cm}^{3}$ con un rango de 181.3 a $486.3 \mathrm{~cm}^{3}$. En los caballos enteros no se observó diferencia estadística entre las diversas medidas de ambos testículos.

Los sementales castrados que mantienen solo el testículo izquierdo presentaron medidas ligeramente mayores que aquellos que solo mantienen el testículo derecho, pero estas diferencias no fueron estadísticamente significativas. El alto, ancho y volumen fue significativamente mayor en los sementales castrados unilateralmente en comparación con los sementales enteros $(\mathrm{p}<0.05)$, especialmente para el testículo izquierdo $(\mathrm{p}<0.0001)$.

La PED estimada en caballos enteros fue de $7.67 \pm 2.32 \times 10^{9}$ espermatozoides (rango de 3.39 a $10.91 \times 10^{9}$ ) y en castrados unilateralmente fue de $5.13 \pm 1.84 \times 10^{9}$ espermatozoides (rango de 2.21 a $8.17 \times 10^{9}$ ). Este valor fue $33 \%$ menor en sementales castrados unilateralmente $(\mathrm{p}<0.0001)$. 
Cuadro 1. Promedio, desviación estándar (de) y rango de las medidas testiculares en sementales enteros de raza Caballo Chileno $(n=31)$

\begin{tabular}{lcccccc}
\hline \multirow{2}{*}{$\begin{array}{l}\text { Medidas } \\
\text { testiculares }\end{array}$} & \multicolumn{2}{c}{ Testículo derecho } & \multicolumn{2}{c}{ Testículo izquierdo } & \multicolumn{2}{c}{ Ambos testículos } \\
\cline { 2 - 7 } & Prom. \pm de & Rango & Prom. \pm de & Rango & Prom. \pm de & Rango \\
\hline Alto $(\mathrm{cm})$ & $6.4 \pm 0.8$ & $4.8-8.2$ & $6.2 \pm 0.7$ & $5.0-7.5$ & $6.3 \pm 0.8$ & $4.8-8.2$ \\
Ancho $(\mathrm{cm})$ & $5.2 \pm 0.9$ & $3.4-7.6$ & $5.3 \pm 0.8$ & $3.6-6.6$ & $5.2 \pm 0.9$ & $3.4-7.6$ \\
Largo $(\mathrm{cm})$ & $9.9 \pm 1.2$ & $7.1-11.9$ & $10.0 \pm 1.1$ & $7.1-12.1$ & $10.0 \pm 1.2$ & $7.1-12.1$ \\
Volumen $\left(\mathrm{cm}^{3}\right)$ & $176.1 \pm 58.2$ & $85-329$ & $175.1 \pm 48.5$ & $85-279$ & $175.6 \pm 53.1$ & $85-329$ \\
\hline
\end{tabular}

Cuadro 2. Promedio, desviación estándar (de) y rango de las medidas testiculares en sementales de raza Caballo Chileno castrados unilateralmente $(n=30)$

\begin{tabular}{lcccc}
\hline \multirow{2}{*}{$\begin{array}{l}\text { Medidas } \\
\text { testiculares }\end{array}$} & \multicolumn{2}{c}{ Testículo derecho } & \multicolumn{2}{c}{ Testículo izquierdo } \\
\cline { 2 - 5 } & Prom. \pm de & Rango & Prom. \pm de & Rango \\
\hline Alto $(\mathrm{cm})$ & $7.1 \pm 1.0$ & $5.4-8.8$ & $7.4 \pm 1.2$ & $5.3-9.1$ \\
Ancho $(\mathrm{cm})$ & $6.2 \pm 0.8$ & $4.6-7.4$ & $6.4 \pm 0.9$ & $4.6-7.6$ \\
Largo $(\mathrm{cm})$ & $10.0 \pm 1.0$ & $8.3-11.8$ & $10.1 \pm 1.0$ & $8.7-12.3$ \\
Volumen $\left(\mathrm{cm}^{3}\right)$ & $234.8 \pm 76.6$ & $123.8-381.7$ & $252.8 \pm 78.3$ & $131.8-372.2$ \\
\hline
\end{tabular}

\section{Discusión}

Las medidas testiculares del Caballo Chileno entero, que por su alzada debería ser considerado un poni, son superiores a las reportadas para razas tipo ponis como el Caballo Chilote (Cox, 2005) o caballos miniatura (Metcalf et al., 1997; Paccamonti et al., 1999). Incluso mayores a las reportadas para caballos frisones (Stout y Colenbrander, 2011), caballos estonianos (Kavak et al., 2003) y para los promedios reportados por Love $e t$ al. $(1990,1991)$ en ponis, caballos livianos y caballos de sangre templada. De hecho, el largo testicular es similar al reportado en sementales de Sangre Templada Holandés (Parlevliet et al., 1994) o caballos pesados menores a 4 años (Stout y Colenbrander, 2011). Evidentemente, la proporción entre el tamaño testicular y el peso corporal sería mayor en los sementales de raza Caballo Chileno que la sugerida por Kavak et al. (2003) y Chenier (2007).

El amplio rango observado en todas las medidas testiculares se puede explicar por la diversidad de edades de los sementales evaluados (de 3 a 20 años), dado que el tamaño testicular aumenta hasta los 6 años (Thompson et al., 1979; Pickett et al., 1981) e incluso hasta más de 10 años según Stout y Colenbrander (2011). Es conocido que el tamaño testicular está influenciado por la edad (Crabtree, 2010) y el tamaño corporal (Kavak et al., 2003; Chenier, 2007). 
En los sementales castrados unilateralmente, el VT fue $39.8 \%$ mayor que en los sementales enteros, influenciado principalmente por el alto y ancho de los testículos. Esta hipertrofia testicular compensatoria del testículo residual ha sido demostrada en caballos (Hoagland et al., 1986) y carneros castrados unilateralmente (Mirando et al., 1989). De hecho, las medidas testiculares de los sementales castrados unilateralmente de este estudio fueron muy superiores a las de ponis, caballos livianos, frisones, caballos de sangre templada y caballos pesados menores a 4 años (Paccamonti et al., 1999; Kavak et al., 2003; Stout y Colenbrander, 2011), siendo muy similares a las medidas testiculares de sementales Tori, cuyo peso corporal promedio es de $660 \mathrm{~kg}$ (Kavak et al., 2003) y de caballos pesados menores a 10 años (Stout y Colenbrander, 2011).

La PED promedio estimada para sementales enteros fue mayor a la reportada en ponis (Paccamonti et al., 1999; Cox, 2005) y caballos livianos (Thompson et al., 1979; Love et al., 1991; Kavak et al., 2003) ya que en que ninguno supera los $5 \times 10^{9}$ espermatozoides al día. El PED obtenido en el Caballo Chileno solo ha sido superada por una raza de mayor peso como Tori (Kavak et al., 2003). En forma similar, la PED promedio en los caballos castrados unilateralmente fue mayor a valores reportados en ponis (Paccamonti et al., 1999; Cox, 2005) y en caballos livianos con ambos testículos (Gebauer et al., 1974a; Thompson et al., 1979; Kavak et al., 2003).

Los resultados permiten concluir que las medidas testiculares de los sementales de raza Caballo Chileno son mayores a las esperadas para su tamaño corporal.

\section{Literatura Citada}

1. Arms R. 2015. The Chilean Horse: America's oldest cowhorse. [Internet] Disponible en: http://www.chilean- horse.com/index.php?option $=\mathrm{com}_{-}$ content\& task= category\&sectionid= $5 \& \mathrm{id}=16 \&$ Itemid $=59$

2. Blanchard TL, Brinsko SP, Love CC, Varner DD. 2008. How to use testicular measurements for first-season subfertility insurance consideration in thoroughbred stallions. LIV Annual Convention AAEP. San Diego, USA: American Association Equine Practitioners.

3. Carroll CL, Huntington PJ. 1988. Body condition scoring and weight estimation of horses. Equine Vet J 20: 41-45.

4. Chenier TS. 2007. Anatomy and examination of the normal testicle. In: Samper JC, Pycock JF, McKinnon AO (eds). Current therapy in equine reproduction. Saint Louis, USA: Saunders Elsevier. p 167-170.

5. Cox AF. 2005. Caracterización andrológica de potros de raza chilota. Tesis de Médico Veterinario. Valdivia: Universidad Austral de Chile. 28 p.

6. Crabtree J. 2010. Prebreeding examination of the stallion. 1. Physical examination. Practice 32: 22-28. doi: 10.1136/inp.b5503

7. Federación de Rodeo Chileno. 2015. Reglamento rodeo chileno. [Internet]. Disponible en: http://www.caballoyrodeo.cl/portal_rodeo/site/edic/base/port/ reglamentos.html

8. García ML, Cabezas I, Guzmán R, Valenzuela S, Merino V, Pérez R. 1997. Características hipométricas, peso corporal y capacidad de carga del caballo fina sangre chileno en rodeo. Av Cien Vet 12: 45-52. doi: 10.5354/07195273.1997.4789

9. Gebauer MP, Pickett BW, Swierstra EE. 1974a. Reproductive physiology of the stallion. II. Daily production and output sperm. J Anim Sci 39: 732-736.

10. Gebauer MP, Pickett BW, Voss JL, Swierstra EE. 1974b. Reproductive physiology of the stallion: daily sperm output and testicular measurements. J Am Vet Med Assoc 165: 711-713. 
11. Hernández RS, Fernández CC, Baptista PL. 2010. Metodología de la investigación. $5^{\mathrm{a}}$ ed. México: McGraw Hill. $368 \mathrm{p}$.

12. Hoagland TA, Mannen KA, Dinger JE, Ott KM, Woody CO, Riesen JW, Daniels W. 1986. Effects of unilateral castration on morphological characteristics of the testis in one-, two, and threeyear old stallions. Theriogenology 26 : 397-405.

13. Kavak A, Lundeheim N, Aidnik M, Einarsson S. 2003. Testicular measurements and daily sperm output of Tori and Estonian breed stallions. Reprod Domest Anim 38: 167-169. doi: 10.1046/j.14390531.2003.00418.x

14. Love CC. 2007. Reproductive examination of the stallion: evaluation of potential breeding soundness. In: Youngquist RS, Threlfall WR (eds). Current therapy in large animal theriogenology. $2^{\text {nd }}$ ed. Saint Louis: Saunders Elsevier. p 10-14.

15. Love CC, García MC, Riera FR, Kenney RM. 1990. Use of testicular volume to predict daily sperm output in the stallion. In: XXXVI Annual Conventions AAEP. Lexington: American Association Equine Practitioners.

16. Love CC, García MC, Riera FR, Kenney RM. 1991. Evaluation of measures taken by ultrasonography and caliper to estimate testicular volume and predict daily sperm output in the stallion. J Reprod Fert (Suppl 44): 99-105.

17. Metcalf ES, Ley WB, Love CC. 1997. Semen parameters of the American miniature horse stallion. In: XLIII Annual Convention AAEP. Phoenix, USA: American Association Equine Practitioners.

18. Mirando MA, Hoagland TA, Woody Jr CO, Riesen JW. 1989. The influence of unilateral castration of testicular morphology and function in adult rams. Biol Reprod 41: 798-806. doi: 10.1095/ biolreprod41.5.798

19. Muñoz L, Ortiz D, Ortiz R, Cabezas I, Briones M. 2012. Determinación de la carga al dorso en caballos de rodeo chile- no y comparación con la capacidad máxima de carga al dorso estimada, de acuerdo a la fórmula de Beltrán (1954). Arch Med Vet 44: 285-289. doi: 10.4067/ S0301-732X2012000300012

20. Paccamonti DL, Buiten A, Parlevliet J, Colenbrander B. 1999. Reproductive parameters of miniature stallions. Theriogenology 51: 1343-1349. doi: 10.1016/S0093-691X(99)00078-3

21. Parlevliet JM, Kemp B, Colenbrander B. 1994. Reproductive characteristics and semen quality in maiden Dutch warmblood stallions. J Reprod Fert 101: 183-187.

22. Pickett BW, Voss JL, Squires EL, Amann RP. 1982. Management of the stallion for maximum reproductive efficiency. USA: Colorado State University Station. General Series $N^{\circ}$ $1005.84 \mathrm{p}$.

23. Porte E, Mansilla A, Pinochet R. 2000. Evolución del nivel de consanguinidad y grado de parentesco de la raza caballar criolla chilena en el periodo 1893-1997. Av Prod Anim 25: 69-76.

24. Sertich PL. 2011. Examination of external genitalia. In: McKinnon AO, Squires EL, Vaala WE, Varner DD (eds). Equine reproduction. Vol 1. $2^{\text {nd }}$ ed. Chichister: Wiley-Blackwell. p 14581461.

25. Stout T, Colenbrander B. 2011. Reproductive parameters of draft horse, Friesian and warmblood stallions. In: McKinnon AO, Squires EL, Vaala WE, Varner DD (eds). Equine reproduction. Vol 1. $2^{\text {nd }}$ ed. Chichister: WileyBlackwell. p 1362-1366.

26. Thompson DL, Pickett BW, Squires EL, Amann RP. 1979. Testicular measurements and reproductive characteristics in stallions. J Reprod Fert (Suppl 27): 13-17.

27. Varner DD. 2011. Evaluation the stallion. What does the practitioner need to know? XII International Congress WEBA. Hyderabad: World Equine Veterinary Association. 